\title{
Avaliação da capacidade funcional dos membros superiores por meio do TEMPA: Valores de referência, efeito da idade, gênero, dominância e relação com a destreza
}

\author{
Upper extremity evaluation test through TEMPA: Reference values, age, \\ gender, dominance effect and relation to dexterity
}

\author{
S.M. Michaelsen, A.C. Ovando, M.A. Natalio, G.Z. Mazo, L.C. Rodrigues
}

\begin{abstract}
RESUMO
Os objetivos do estudo foram verificar o efeito da idade, gênero e dominância na capacidade funcional dos membros superiores (MMSS), desenvolver valores de referência para a versão brasileira do TEMPA (Test d'Evaluation de la performance des Membres supérieurs dês Personnes Agées; teste de função dos MMSS) e verificar a relação entre a destreza digital e manual e a capacidade funcional dos MMSS. Participaram 281 indivíduos acima de 40 anos. As destrezas manual e digital foram avaliadas, respectivamente, através do Box and Blocks Test (BBT) e do Nine Hole Peg Test (NHPT). A ANOVA foi utilizada para avaliar o efeito da idade, do sexo e lateralidade. O coeficiente de correlação de Pearson foi usado para avaliar a relação entre a destreza e o TEMPA. Nas faixas etárias de 70-79 e acima dos 80 anos observou-se um declínio nas tarefas do TEMPA comparativamente às faixas etárias inferiores. Não foram observadas diferenças significativas entre as faixas etárias de 40-49, 50-59 e 60-69 anos, em nenhuma das tarefas. O declínio da destreza digital nas mulheres e da destreza manual nos homens apresentou correlação com a diminuição da função dos MMSS. Conhecer os efeitos do envelhecimento na função dos MMSS e o impacto das alterações de destreza é importante para identificar mudanças patológicas que afetam os MMSS.
\end{abstract}

Palavras-chave: função dos membros superiores, destreza, envelhecimento

The purpose of this study was to verify the effect of age, gender and dominance on upper limb functional capacity and develop reference values on Functional performance test for upper extremity, Brazilian version of TEMPA (Test d'Evaluation de la performance des Membres supérieurs dês Personnes Agées), and verify the relationship between hand and digital dexterity and arm functional capacity. Participated in the study 281 individuals, aging more than 40 years old. Manual dexterity and finger dexterity were assessed with Box and Blocks Test (BBT) and Nine Hole Peg Test, respectively. ANOVA evaluated the effects of age in men and women and effects of gender and laterality. Pearson`s correlation evaluated the relation between dexterity and upper limb function. In 70 to 79 years strata and the strata older than 80 TEMPA tasks have shown a decline compared to the younger strata. No significant differences were see between 40-49, 50-59 e 60-69 years old strata for any task. Decline in digital dexterity in women and in hand dexterity in man was correlated with decrease in upper limb function. The knowledge on effects of aging on upper limb function and dexterity will help differentiate between normal and pathological aging in upper extremity performance.

Keywords: upper extremity function, dexterity, aging

Submetido: 15.04.2010 | Aceite: 31.07.2010

Stella Maris Michaelsen, Angélica Cristiane Ovando, Mavie Amaral Natalio, Giovana Zarpellon Mazo e Letícia Cardoso Rodrigues. Programa de Pós-Graduação em Ciências do Movimento Humano da Universidade do Estado de Santa Catarina - UDESC - Florianópolis - SC, Brasil.

Endereço para correspondência: Stella Maris Michaelsen, Programa de Pós-Graduação em Ciências do Movimento Humano - CEFID/UDESC, Laboratório de Controle Motor, Rua Pascoal Simone, 358 - Coqueiros, CEP: 88080-350 Florianópolis - SC, Brasil.

E-mail: michaelsenstella@hotmail.com 
O conhecimento das alterações funcionais que ocorrem com o avanço da idade é necessário para o estabelecimento de cuidados adequados e ações preventivas visando a população idosa. Em relação aos efeitos do envelhecimento na função dos membros superiores, a maioria dos estudos desenvolvidos no Brasil envolvendo a população idosa tem focado no declínio da força de preensão (Geraldes, Oliveira, Albuquerque, Carvalho, \& Farinatti, 2008). Menor atenção tem sido dada à destreza manual e digital que também sofrem um declínio com a idade (Desrosiers, Bravo, Hébert, Dutil, \& Mercier, 1994; Kellor, Frost, Silberberg, Iversen, \& Cummings, 1971) e podem estar relacionadas com uma queda na capacidade funcional dos membros superiores (MMSS) (Desrosiers, Hébert, Bravo, \& Dutil, 1995), afetando a independência na realização de atividades de vida diária (AVDs).

Um dos primeiros testes padronizados para avaliar a função dos membros superiores foi o teste de função da mão de Jebsen (Jebsen, Taylor, Trieschmann, Trotter, \& Howard, 1969), que apresenta uma série de sete subtestes representativos de funções executadas com as mãos. Embora este teste seja bastante utilizado para avaliar a capacidade funcional dos MMSS de idosos, tem como limitação o fato de testar apenas tarefas unilaterais, focar a função manual sem considerar o movimento proximal, e ainda, utilizar tarefas de natureza artificial e pouco ligadas às AVDs (Desrosiers, Hébert, Dutil, \& Bravo, 1993).

Nesse contexto, foi desenvolvido e validado o teste de desempenho funcional dos membros superiores - TEMPA (Test d'Évaluation de la performance des Membres superieurs des Personnes Agées) no Canadá (Desrosiers et al., 1993). Esse teste é composto por nove tarefas padronizadas que simulam AVDs tanto uni quanto bilaterais, e os valores normativos para o tempo de execução das tarefas para a população idosa do Canadá foram publicados em 1995 (Desrosiers et al., 1995).
No Brasil, uma versão adaptada do TEMPA, constituída por quatro tarefas bilaterais e quatro tarefas unilaterais, foi validada para adultos com hemiparesia (Michaelsen, Natalio, Silva, \& Pagnussat, 2008). Neste estudo, a confiabilidade do tempo para execução das tarefas também foi avaliada em 23 indivíduos controles, sem sequelas neurológicas, com média de idade de $60.0 \pm 11.5$ anos. A versão Brasileira do TEMPA apresentou índices adequados de confiabilidade, entretanto, ainda não se tem valores de referência para a população brasileira. Assim, tornam-se importantes estudos que desenvolvam valores de referência para pesquisas com populações especiais e indivíduos que apresentam disfunções dos membros superiores. Além disso, estes valores servirão de parâmetros para a avaliação e intervenções.

Da mesma forma, estudos que avaliam a capacidade funcional dos membros superiores, por meio do TEMPA, e relacionam com a destreza digital e manual com o avançar da idade ainda são carentes no Brasil. Em estudo realizado no Canadá, Desrosiers et al. (1995) avaliaram tanto a destreza manual como a destreza digital em idosos e encontraram uma correlação significativa com a maioria das tarefas do TEMPA.

Sendo assim, o presente estudo teve como objetivos desenvolver valores de referência para a capacidade funcional os membros superiores, por meio do TEMPA, para adultos e idosos do sul do Brasil, em função da faixa etária, sexo e lateralidade, além de avaliar a relação entre a destreza manual e digital e o desempenho funcional do membro superior.

\section{MÉTODO}

\section{Amostra}

Participaram do estudo 281 sujeitos sendo 168 mulheres $(65.0 \pm 10.8$ anos $)$ e 113 homens (62.5 \pm 11.9 anos), estratificados em cinco faixas etárias: 40 a 49; 50 a 59; 60 a 69; 70 a 79; e acima de 80 anos. A distribuição predominante de mulheres $(59.8 \%)$ está de acordo com os dados demográficos atuais, nos 
quais se observa maior presença relativa de mulheres na população idosa em comparação com homens, principalmente nas faixas etárias mais avançadas (IBGE, 2002).

Os participantes do estudo foram recrutados a partir de grupos de convivência, grupos de atividade física, assim como de estabelecimentos públicos de dois municípios do sul do Brasil (Florianópolis - SC e Ijuí RS). Os sujeitos do estudo foram selecionados a partir dos seguintes critérios de inclusão: adultos, residentes na comunidade com 40 anos ou mais, e com capacidade de compreensão de ordens simples e acuidade visual (ou com correção - óculos, lentes). Os indivíduos que residiam em instituições de longa permanência ou que apresentaram alguma patologia neurológica e/ou ortopédica diagnosticada afetando os MMSS foram excluídos da pesquisa.

Com a aprovação do Comitê de Ética em Pesquisa em Seres Humanos da Instituição (Parecer n. ${ }^{\circ}$ 179/2007), o termo de consentimento foi obtido de cada participante.

\section{Instrumentos e Procedimentos}

A capacidade funcional dos MMSS foi avaliada por meio da velocidade de execução das tarefas do TEMPA.

A versão brasileira do TEMPA é composta por quatro tarefas bilaterais (abrir um pote e tirar uma colher cheia de café; destrancar uma fechadura e abrir um recipiente contendo pílulas; escrever em um envelope e colar um selo; embaralhar e distribuir cartas de jogo) e quatro tarefas unilaterais (pegar e mover um pote; pegar uma jarra e servir água em um copo; manusear moedas; pegar e mover pequenos objetos). Cada uma das tarefas é demonstrada, permitindo-se o treino para adaptação quando necessário e em seguida o teste é realizado e cronometrado.

A destreza manual foi avaliada por meio do teste de caixa e blocos (Box and Blocks Test BBT) (Mathiowetz, Volland, Kashman, \& Weber, 1985), o qual consiste em transportar o maior número possível de pequenos cubos de madeira de um compartimento a outro de uma caixa durante 60 segundos.

A destreza digital foi avaliada por meio do teste dos "nove pinos e nove buracos" (Nine Hole Peg Test - NHPT) (Mathiowetz, Weber, Kashman, \& Volland, 1985), o qual consiste em verificar o tempo necessário para colocar e retirar nove pinos de madeira em nove orifícios. No presente estudo optamos por utilizar a "abreviação" em inglês, considerando a grande divulgação dos testes como "BBT" e "NHPT".

Os testes BBT e NHPT foram utilizados na população brasileira por Mendes, Tilbery, Balsimelli, Moreira e Cruz (2001) e Tilbery et al. (2005), em estudos com indivíduos com diagnóstico de esclerose múltipla e adultos saudáveis.

\section{Análise Estatística}

Todas as análises estatísticas foram conduzidas no programa SPSS, versão 17.0.

Foram calculadas as médias das variáveis TEMPA (segundos), BBT (número de blocos) e NHPT (segundos).

Para as tarefas bilaterais, foi realizada a ANOVA two-way para testar o efeito da idade (faixas etárias 40 a 49; 50 a 59; 60 a 69; 70 a 79; e acima de 80 anos) e do sexo (masculino e feminino) na capacidade funcional dos MMSS em cada uma das tarefas do TEMPA.

Para as tarefas unilaterais, foi realizada a ANOVA three-way para testar o efeito da idade, sexo e membro superior (direito e esquerdo) na capacidade funcional dos MMSS em cada uma das tarefas do TEMPA. Quando encontradas diferenças significativas, foi realizado o post-hoc de Tukey a fim de identificar as diferenças entre grupos.

A relação entre a função dos membros superiores (TEMPA) e as variáveis de destreza (BBT e NHPT) foi analisada através do coeficiente de correlação de Pearson.

Em todas as análises foi fixado um nível de significância de 5\% ( $p<.05$ ou IC 95\%). 


\section{RESULTADOS}

Efeito da idade, sexo e membro superior sobre o tempo de execução nas tarefas do TEMPA

A ANOVA two-way apresentou um efeito principal de idade nas tarefas bilaterais de abrir pote e tirar café $\left(F_{(4,271)}=11.47\right)$, destrancar fechadura $\left(F_{(4,271)}=15.51\right)$, escrever e colar selo $\left(F_{(4,271)}=10.28\right)$ e embaralhar cartas $\left(F_{(4,271)}=18.87, p<.001\right.$ para todas as tarefas). As faixas etárias de 70 a 79 e acima de 80 anos apresentaram um desempenho mais lento em todas as tarefas bilaterais comparativamente às faixas etárias mais jovens (post-hoc Tukey com $p<.01$ para todas as comparações). A faixa etária acima de 80 anos também apresentou um desempenho mais lento quando comparada com a faixa etária de
70 a 79 anos $(p<.01$ para todas as tarefas, excetuando-se a tarefa de escrever e colar selo que foi semelhante entre estas duas faixas etárias). A única tarefa bilateral que apresentou efeito principal de sexo $\left(F_{(1,271)}=\right.$ 4.36, $p=.038$ ) foi a tarefa de abrir pote e tirar café, sendo os homens mais lentos para executar esta tarefa. Não foi observado nenhum efeito de interação entre idade e sexo nas tarefas bilaterais (ver Tabela 1).

Com relação às tarefas unilaterais, a ANOVA three-way apresentou efeito principal de idade em todas as tarefas avaliadas: pegar e mover um pote $\left(F_{(4,271)}=5.90\right)$, pegar jarra e servir água $\left(F_{(4,271)}=13.00\right)$, manusear moedas $\left(F_{(4,271)}=11.06\right)$ e pegar e mover pequenos objetos $\left(F_{(4,271)}=12.02, p<.001\right.$ para todas as tarefas).

Tabela 1

Tempo de execução $(M \pm D P)$ das tarefas bilaterais do TEMPA (em segundos) de acordo com faixa etária para os grupos de mulheres e homens

\begin{tabular}{|c|c|c|c|c|c|}
\hline Tarefas do TEMPA & $\begin{array}{c}40-49 \text { anos } \\
(+, n=19) \\
(\delta, n=21)\end{array}$ & $\begin{array}{l}50-59 \text { anos } \\
(+, n=34) \\
(\hat{\jmath}, n=25)\end{array}$ & $\begin{array}{l}60-69 \text { anos } \\
(+, n=50) \\
(\hat{\jmath}, n=31)\end{array}$ & $\begin{array}{l}70-79 \text { anos } \\
(+, n=48) \\
(0, n=27)\end{array}$ & $\begin{array}{c}+80 \text { anos } \\
(+, n=17) \\
(\delta, n=9)\end{array}$ \\
\hline \multicolumn{6}{|l|}{ Mulheres } \\
\hline Abrir pote e tirar café (s) & $9.1 \pm 1.5$ & $8.7 \pm 2.3$ & $8.0 \pm 2.1$ & $9.4 \pm 2.4$ & $12.5 \pm 3.4$ \\
\hline Mínimo - Máximo & $6.4-11.9$ & $5.6-15.1$ & $4.4-13.6$ & $4.5-15.3$ & $7.8-22.2$ \\
\hline Destrancar fechadura e retirar & $11.3 \pm 1.7$ & $11.2 \pm 2.7$ & $11.7 \pm 2.9$ & $13.1 \pm 2.5$ & $16.3 \pm 4.9$ \\
\hline $\begin{array}{l}\text { pilulas (S) } \\
\text { Mínimo - Máximo }\end{array}$ & $8.3-15.3$ & $7.6-17.2$ & $6.8-20.7$ & $7.5-18.9$ & $9.0-25.2$ \\
\hline Escrever e colar selo (s) & $14.5 \pm 5.6$ & $15.8 \pm 7.2$ & $16.0 \pm 7.7$ & $21.7 \pm 10.5$ & $23.3 \pm 9.8$ \\
\hline Mínimo - Máximo & $9.1-28.4$ & $8.7-31.3$ & $8.3-40.1$ & $9.6-49.3$ & $14.4-49.3$ \\
\hline Embaralhar cartas (s) & $15.8 \pm 2.7$ & $17.5 \pm 4.9$ & $17.9 \pm 4.1$ & $22.5 \pm 5.4$ & $27.2 \pm 9.3$ \\
\hline Mínimo - Máximo & $10.3-21.4$ & $9.3-35.5$ & $11.5-32.7$ & $13.0-34.8$ & $18.0-55.5$ \\
\hline \multicolumn{6}{|l|}{ Homens } \\
\hline Abrir pote e tirar café (s) & $9.8 \pm 2.6$ & $9.2 \pm 2.0$ & $9.2 \pm 2.4$ & $11.1 \pm 3.4$ & $12.0 \pm 3.2$ \\
\hline Mínimo - Máximo & $2.2-14.4$ & $6.4-13.7$ & $5-16.2$ & $4.5-20.1$ & $6.6-14.7$ \\
\hline Destrancar fechadura e retirar & $12.5 \pm 2.1$ & $11.0 \pm 2.4$ & $12.6 \pm 3.5$ & $13.7 \pm 3.0$ & $16.2 \pm 4.8$ \\
\hline $\begin{array}{l}\text { pilulas (S) } \\
\text { Mínimo - Máximo }\end{array}$ & $8.5-17.0$ & $7.0-18.0$ & $7.3-20.6$ & $7.6-21.2$ & $11.6-27.7$ \\
\hline Escrever e colar selo (s) & $11.9 \pm 2.4$ & $13.0 \pm 5.4$ & $16.4 \pm 7.2$ & $18.7 \pm 7.7$ & $22.7 \pm 12.4$ \\
\hline Mínimo - Máximo & $8.6-17.3$ & $7.5-33.8$ & $8-37.2$ & $10.0-40.0$ & $13.3-53.7$ \\
\hline Embaralhar cartas (s) & $17.1 \pm 3.2$ & $16.6 \pm 3.0$ & $18.9 \pm 5.1$ & $21.5 \pm 4.9$ & $22.4 \pm 5.4$ \\
\hline Mínimo - Máximo & $11.5-24.3$ & $11.9-22.1$ & $10.5-31.3$ & $11.9-31.1$ & $13.8-31.0$ \\
\hline
\end{tabular}


Tabela 2

Tempo de execução $(M \pm D P)$ das tarefas unilaterais do TEMPA de acordo com faixa etária para os grupos de mulheres e homens

\begin{tabular}{|c|c|c|c|c|c|}
\hline Tarefas do TEMPA & $\begin{array}{l}40-49 \text { anos } \\
(+, n=19) \\
(\lesssim, n=21)\end{array}$ & $\begin{array}{l}50-59 \text { anos } \\
(0, n=34) \\
(\hat{\jmath}, n=25)\end{array}$ & $\begin{array}{l}60-69 \text { anos } \\
(0, n=50) \\
(\delta, n=31)\end{array}$ & $\begin{array}{l}70-79 \text { anos } \\
(0, n=48) \\
(\hat{0}, n=27)\end{array}$ & 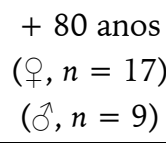 \\
\hline \multicolumn{6}{|l|}{ Mulheres } \\
\hline $\begin{array}{l}\text { Pegar e mover pote } \\
\text { MS D } \\
\text { Mínimo - Máximo MS D }\end{array}$ & $\begin{array}{l}1.9 \pm .3 \\
1.3-2.4\end{array}$ & $\begin{array}{l}1.9 \pm .5 \\
1.2-3.7\end{array}$ & $\begin{array}{l}1.7 \pm .4 \\
1.1-3.3\end{array}$ & $\begin{array}{l}1.8 \pm .3 \\
1.2-2.7\end{array}$ & $\begin{array}{l}2.2 \pm .7 \\
1.4-2.2\end{array}$ \\
\hline $\begin{array}{l}\text { MS E } \\
\text { Mínimo - Máximo MS E }\end{array}$ & $\begin{array}{c}1.9 \pm .3 \\
1.5-2.35\end{array}$ & $\begin{array}{c}1.9 \pm .5 \\
1.12-3.2\end{array}$ & $\begin{array}{l}1.7 \pm .3 \\
1.2-3.0\end{array}$ & $\begin{array}{l}1.8 \pm .3 \\
1.1-2.5\end{array}$ & $\begin{array}{l}2.3 \pm .6 \\
1.6-3.7\end{array}$ \\
\hline $\begin{array}{l}\text { Pegar jarra e servir água } \\
\text { MS D } \\
\text { Mínimo - Máximo MS D }\end{array}$ & $\begin{array}{c}8.4 \pm .9 \\
6.2-10.0\end{array}$ & $\begin{array}{c}8.6 \pm 1.5 \\
5.9-11.7\end{array}$ & $\begin{array}{l}8.5 \pm 1.2 \\
6.4-11.4\end{array}$ & $\begin{array}{l}9.4 \pm 1.6 \\
6.0-13.0\end{array}$ & $\begin{array}{l}10.8 \pm 1.8 \\
7.2-14.5\end{array}$ \\
\hline $\begin{array}{l}\text { MS E } \\
\text { Mínimo - Máximo MS E }\end{array}$ & $\begin{array}{c}8.4 \pm .9 \\
6.9-10.4\end{array}$ & $\begin{array}{c}8.3 \pm 1.6 \\
5.8-11.7\end{array}$ & $\begin{array}{c}8.2 \pm 1.4 \\
5.8-11.5\end{array}$ & $\begin{array}{c}9.1 \pm 1.2 \\
5.8-11.9\end{array}$ & $\begin{array}{l}11.2 \pm 2.2 \\
7.7-14.8\end{array}$ \\
\hline $\begin{array}{l}\text { Manusear moedas } \\
\text { MS D } \\
\text { Mínimo - Máximo MS D }\end{array}$ & $\begin{array}{c}7.7 \pm 1.3 \\
5.3-10.3\end{array}$ & $\begin{array}{c}8.1 \pm 1.6 \\
5.3-12.9\end{array}$ & $\begin{array}{c}7.7 \pm 1.8 \\
5.6-17.1\end{array}$ & $\begin{array}{c}8.3 \pm 1.6 \\
5.6-13.1\end{array}$ & $\begin{array}{l}10.0 \pm 2.0 \\
6.9-13.4\end{array}$ \\
\hline $\begin{array}{l}\text { MS E } \\
\text { Mínimo - Máximo MS E }\end{array}$ & $\begin{array}{c}8.3 \pm 1.7 \\
5.9-13.6\end{array}$ & $\begin{array}{c}8.2 \pm 1.5 \\
5.4-11.6\end{array}$ & $\begin{array}{c}8.1 \pm 1.3 \\
6.2-13.5\end{array}$ & $\begin{array}{c}9.0 \pm 1.8 \\
5.8-16.2\end{array}$ & $\begin{array}{l}10.8 \pm 2.3 \\
6.9-14.9\end{array}$ \\
\hline $\begin{array}{l}\text { Pegar e mover pequenos objetos } \\
\text { MS D } \\
\text { Mínimo - Máximo MS D }\end{array}$ & $\begin{array}{c}6.3 \pm .9 \\
4.7-7.9\end{array}$ & $\begin{array}{l}6.4 \pm 1.3 \\
4.1-9.9\end{array}$ & $\begin{array}{c}6.3 \pm 1.3 \\
4.1-12.9\end{array}$ & $\begin{array}{c}7.1 \pm 1.4 \\
4.7-13.1\end{array}$ & $\begin{array}{l}8.6 \pm 1.8 \\
5.9-12.1\end{array}$ \\
\hline $\begin{array}{l}\text { MS E } \\
\text { Mínimo - Máximo MS E }\end{array}$ & $\begin{array}{l}6.4 \pm 1.0 \\
5.0-9.0\end{array}$ & $\begin{array}{c}6.6 \pm 1.7 \\
4.4-13.5\end{array}$ & $\begin{array}{l}6.5 \pm 1.3 \\
4.7-11\end{array}$ & $\begin{array}{l}7.2 \pm 1.1 \\
5.0-9.9\end{array}$ & $\begin{array}{c}8.7 \pm 2.2 \\
6.0-14.0\end{array}$ \\
\hline \multicolumn{6}{|l|}{ Homens } \\
\hline $\begin{array}{l}\text { Pegar e mover pote } \\
\text { MS D } \\
\text { Mínimo - Máximo MS D }\end{array}$ & $\begin{array}{l}1.7 \pm .3 \\
1.0-2.3\end{array}$ & $\begin{array}{c}1.8 \pm .6 \\
1.2-3.9\end{array}$ & $\begin{array}{l}1.6 \pm .4 \\
1.0-2.5\end{array}$ & $\begin{array}{c}1.8 \pm .4 \\
1.1-2.9\end{array}$ & $\begin{array}{l}2.1 \pm .2 \\
1.6-2.3\end{array}$ \\
\hline $\begin{array}{l}\text { MS E } \\
\text { Mínimo - Máximo MS E }\end{array}$ & $\begin{array}{c}1.7 \pm .4 \\
1.1-2.5\end{array}$ & $\begin{array}{c}1.8 \pm .5 \\
1.3-3.5\end{array}$ & $\begin{array}{c}1.7 \pm .3 \\
1.2-2.3\end{array}$ & $\begin{array}{c}1.9 \pm .5 \\
1.2-3.1\end{array}$ & $\begin{array}{l}2.0 \pm .5 \\
1.2-2.7\end{array}$ \\
\hline $\begin{array}{l}\text { Pegar jarra e servir água } \\
\text { MS D } \\
\text { Mínimo - Máximo MS D }\end{array}$ & $\begin{array}{c}9.2 \pm 1.1 \\
7.6-11.8\end{array}$ & $\begin{array}{c}9.2 \pm 1.6 \\
6.4-13.2\end{array}$ & $\begin{array}{c}9.7 \pm 1.4 \\
6.7-12.3\end{array}$ & $\begin{array}{c}10.3 \pm 1.7 \\
7.1-14\end{array}$ & $\begin{array}{l}10.2 \pm 2.1 \\
7.8-14.5\end{array}$ \\
\hline $\begin{array}{l}\text { MS E } \\
\text { Mínimo - Máximo MS E }\end{array}$ & $\begin{array}{l}9.1 \pm 1.0 \\
7-11.5\end{array}$ & $\begin{array}{c}9.2 \pm 1.4 \\
7.0-12.1\end{array}$ & $\begin{array}{c}9.3 \pm 1.4 \\
6.8-12.2\end{array}$ & $\begin{array}{l}10.6 \pm 1.8 \\
7.2-13.3\end{array}$ & $\begin{array}{l}10.5 \pm 2.9 \\
7.0-15.1\end{array}$ \\
\hline $\begin{array}{l}\text { Manusear moedas } \\
\text { MS D } \\
\text { Mínimo - Máximo MS D }\end{array}$ & $\begin{array}{c}7.9 \pm .9 \\
5.8-9.1\end{array}$ & $\begin{array}{c}7.7 \pm 1.5 \\
5.9-12.1\end{array}$ & $\begin{array}{c}8.4 \pm 1.6 \\
5.6-12.1\end{array}$ & $\begin{array}{c}9.0 \pm 1.4 \\
6.3-14.3\end{array}$ & $\begin{array}{l}9.9 \pm 2.1 \\
6.0-12.8\end{array}$ \\
\hline $\begin{array}{l}\text { MS E } \\
\text { Mínimo - Máximo MS E }\end{array}$ & $\begin{array}{c}8.4 \pm 1.2 \\
6.3-11.3\end{array}$ & $\begin{array}{c}8.1 \pm 1.6 \\
6.3-12.3\end{array}$ & $\begin{array}{c}8.6 \pm 1.6 \\
5.7-13.0\end{array}$ & $\begin{array}{c}9.8 \pm 2.0 \\
7.2-13.1\end{array}$ & $\begin{array}{l}10.0 \pm 2.1 \\
7.3-14.2\end{array}$ \\
\hline $\begin{array}{l}\text { Pegar e mover pequenos objetos } \\
\text { MS D } \\
\text { Mínimo - Máximo MS D }\end{array}$ & $\begin{array}{c}6.7 \pm .8 \\
5.1-8.1\end{array}$ & $\begin{array}{l}6.6 \pm 1.3 \\
4.6-9.7\end{array}$ & $\begin{array}{c}7.2 \pm 1.4 \\
5.0-10.4\end{array}$ & $\begin{array}{c}7.7 \pm 1.7 \\
5.4-12.2\end{array}$ & $\begin{array}{l}8.4 \pm 1.7 \\
6.1-11.2\end{array}$ \\
\hline $\begin{array}{l}\text { MS E } \\
\text { Mínimo - Máximo MS E }\end{array}$ & $\begin{array}{l}7.0 \pm .8 \\
5.6-8.4\end{array}$ & $\begin{array}{c}7.0 \pm 1.7 \\
4.6-12.6\end{array}$ & $\begin{array}{c}7.0 \pm 1.6 \\
4.6-10.7\end{array}$ & $\begin{array}{c}7.9 \pm 2.0 \\
4.9-14.7\end{array}$ & $\begin{array}{l}8.3 \pm 1.4 \\
6.8-11.8\end{array}$ \\
\hline
\end{tabular}

Nota: MS D - Membro Superior Direito; MS E - Membro Superior Esquerdo 
A faixa etária acima de 80 anos apresentou um desempenho mais lento em todas as tarefas unilaterais comparativamente às faixas etárias mais jovens (post-hoc Tukey com $p<.001$ para todas as comparações). O estrato de 70 a 79 anos também foi mais lento que as faixas etárias mais jovens, excetuando-se a tarefa pegar e mover um pote $(p<.01$ para todas as comparações). Os homens foram mais lentos que as mulheres nas tarefas pegar a jarra e servir água e pegar e mover pequenos objetos, com um efeito principal de sexo, $F_{(1,271)}=$ 12.67, $p<.001$ e $F_{(1,271)}=5.05, p=.025$, respectivamente. Nas tarefas manusear moedas e pegar pequenos objetos, foi observado efeito principal de MMSS, $F_{(1,271)}=25.54, p<.001 \mathrm{e}$ $F_{(1,271)}=4.03, p=.04$, respectivamente, sendo que os participantes realizaram estas tarefas mais rapidamente com o braço direito (ver Tabela 2). Não foram observadas interações entre idade e sexo, entre idade e MMSS, entre sexo e MMSS ou ainda entre idade, sexo e MMSS nas tarefas unilaterais.

\section{Correlações entre a destreza manual e digital e a capacidade funcional dos membros superiores}

Considerando que escore do BBT é expresso em número de blocos (quanto maior o número de blocos, mais rápido é o participante) e o tempo para execução das tarefas do TEMPA é expresso em segundos, as correlações entre destreza manual e o TEMPA foram negativas. Já em relação à destreza digital, como o escore do NHPT é também em segundos, as correlações positivas entre o NHPT e o TEMPA indicam que quanto maior o tempo gasto para realizar a tarefa de destreza digital, maior é o tempo gasto para concluir a tarefa do TEMPA. Como esperado, as tarefas unilaterais de manusear moedas e pegar e mover pequenos objetos apresentaram correlação com a destreza digital para ambas as mãos.

Nas tarefas que apresentaram um efeito significativo de sexo, a fim de identificar uma eventual associação entre a destreza e o declínio na capacidade funcional dos MMSS, a análise da relação entre a destreza manual e digital e o desempenho no TEMPA foi realizada considerando separadamente os participantes do sexo feminino e do sexo masculino (ver Tabela 3). Nas demais tarefas, a análise foi feita para o grupo todo (homens e mulheres). As tarefas bilaterais de destrancar uma fechadura e de embaralhar e distribuir cartas apresentaram uma correlação variando entre .43 e $.49(p<.05)$ com a destreza manual e entre .46 e $.54(p<.01)$ com a destreza digital. A correlação entre a destreza manual e digital e a tarefa de pegar e mover pote foi baixa $(r \leq .30, p<.05)$. A correlação da destreza manual e digital com a tarefa de manusear moedas foi respectivamente de $.37 \mathrm{e}$ $.42(p<.01)$ para o membro superior direito e

Tabela 3

Correlação entre o tempo de execução nas tarefas do TEMPA e o desempenho da destreza manual (BBT) e digital (NHPT), em homens e mulheres

\begin{tabular}{lcccc}
\multicolumn{1}{c}{ Tarefas } & \multicolumn{2}{c}{ Homens } & \multicolumn{2}{c}{ Mulheres } \\
& BBT & NHPT & BBT & NHPT \\
\hline $\begin{array}{l}\text { Abrir pote e tirar café } \\
\text { Pegar jarra e servir água }\end{array}$ & & $.32^{*}$ & & $.51^{*}$ \\
MS D & $-.34^{*}$ & $.37^{*}$ & $-.42^{*}$ & $.46^{*}$ \\
MS E & $-.39^{*}$ & $.35^{*}$ & $-.39^{*}$ & $.41^{*}$ \\
Pegar e mover pequenos objetos & & & & $.60^{*}$ \\
MS D & $-.55^{*}$ & $.47^{*}$ & $-.38^{*}$ & $.55^{*}$ \\
MS E & $-.47^{*}$ & $.44^{*}$ & $-.39^{*}$ &
\end{tabular}

${ }^{*} p \leq .01 ;$ Nota: BBT - Box and blocks test; NHPT - Nine hole peg test 
de .36 e $.35(p<.01)$ para o membro superior esquerdo. Os valores de correlação entre a destreza digital e o desempenho nas tarefas de abrir um pote e tirar café, pegar jarra e servir água e pegar e mover pequenos objetos foram maiores nas mulheres comparativamente aos homens, já em relação à destreza manual e a tarefa de mover pequenos objetos foi maior para os homens (ver Tabela 3).

\section{DISCUSSÃO}

O presente estudo buscou avaliar a capacidade funcional dos membros superiores de adultos e idosos do sul do Brasil, em função da faixa etária, sexo e lateralidade, além de avaliar a relação entre a destreza manual e digital e o desempenho funcional do membro superior.

Nossos resultados corroboram com o estudo de Desrosiers et al. (1995), no qual o tempo para a execução das tarefas do TEMPA aumentou significativamente com a idade. Além disso, um estudo longitudinal avaliando 264 indivíduos idosos por um período de três anos observou diferenças significativas no desempenho de todas as tarefas do TEMPA, com exceção da tarefa de mover uma jarra com a mão esquerda, demonstrando o efeito do envelhecimento sobre o desempenho funcional dos membros superiores (Desrosiers, Hébert, Bravo, \& Rochette, 1999). Entretanto, as perdas não foram lineares e o grupo mais idoso apresentou perdas mais significativas. Hackel, Wolfe, Bang e Canfield (1992) avaliaram a função do membro superior em 121 idosos de 60 a 89 anos através do teste de Jebsen, observaram que a função do membro superior decresce com o avançar da idade, tanto em homens, quanto em mulheres. Entretanto, como descrito anteriormente, o teste de Jebsen avalia apenas tarefas unilaterais e de pequena complexidade, sem avaliar tarefas que exijam uma sequência de movimentos como as tarefas propostas no TEMPA.

Em idosos, os maiores declínios da função dos membros superiores estão relacionados com fatores neurológicos como a diminuição da força, da velocidade dos movimentos braçomão e da sensação vibratória (Polvin, Syndulcko, Tourtellote, Lemmon, \& Polvin, 1980). É descrito na literatura que a função manual permanece estável até à idade de 65 anos, depois da qual diminui progressivamente. Como foi observado no estudo de Shiffman (1992), a diferença no desempenho relacionado com a idade no padrão de preensão, força, tempo de desempenho e amplitude de movimento torna-se mais aparente a partir de 75 anos. Nossos resultados confirmam que o maior declínio da função dos membros superiores é observado a partir dos 70 anos de idade, em ambos os sexos.

Semelhante ao estudo de Desrosiers et al. (1995), esta pesquisa encontrou uma diferença no desempenho que foi dependente do sexo, visto que as mulheres apresentaram um melhor desempenho nas tarefas de abrir pote e tirar café e pegar e servir água. Além disso, as mulheres também apresentaram um melhor desempenho na tarefa envolvendo destreza digital (pegar e mover pequenos objetos). As diferenças no desempenho de tarefas dos MMSS ligadas ao sexo são mais evidentes em tarefas que exigem habilidades com os dedos; a literatura sugere que não existe diferença importante ligada ao sexo nas tarefas de destreza manual, avaliadas pelo BBT (Desrosiers et al., 1994; Mathiowetz et al., 1985). Em um estudo recente com o teste de Moberg, o qual apresenta alguma semelhança com a tarefa mover pequenos objetos do TEMPA, as mulheres apresentaram um melhor desempenho em recolher os pequenos objetos de metais utilizados no teste (Amirjani, Ashworth, Gordon, Edwards, \& Chan, 2007). Entretanto, nossos resultados mostraram que as mulheres apresentaram, também, melhor desempenho na tarefa bilateral de abrir um pote e tirar uma colher cheia de café. Isto pode ser explicado pelas tarefas domésticas serem desempenhadas preferencialmente pelas mulheres em nossa cultura. No estudo de Desrosiers et al. (1995), os homens foram mais rápidos que as mulheres na tarefa de 
mover uma jarra, resultado não encontrado no presente estudo.

As tarefas unilaterais, de maior destreza, foram as que apresentaram diferenças significativas entre o membro superior direito e esquerdo. Estes resultados são semelhantes aos encontrados no estudo de Amirjani et al. (2007), no qual o desempenho no teste de Moberg (parte que avalia a motricidade fina) foi melhor no membro superior direito. Assim como no estudo de Desrosiers et al. (1995), o desempenho do membro superior direito foi superior nas tarefas de manusear moedas e mover pequenos objetos, confirmando assim que o efeito de dominância é mais evidente para tarefas que exigem maior destreza.

Neste estudo, verificou-se que com o avanço da idade ocorre um aumento no tempo de execução das tarefas do TEMPA, achado que corrobora com outras pesquisas (Desrosiers et al., 1995). Além disso, tanto a destreza manual (BBT), quanto a destreza digital (NHPT), correlacionaram-se com a capacidade funcional dos MMSS (TEMPA).

O estudo de Desrosiers et al. (1995), também mostrou haver uma relação entre a capacidade funcional dos membros superiores e a destreza digital e manual dos participantes, independente do sexo. Em seu estudo, todas as tarefas do TEMPA apresentaram correlações significativas com a destreza manual, com valores de coeficiente de Pearson de no mínimo .48 na tarefa de pegar e mover uma jarra, e máximo de $.63 \mathrm{com}$ a tarefa de abrir um pode e tirar café. Da mesma forma, todas as tarefas do TEMPA apresentaram correlação significativa com a destreza digital, sendo as maiores correlações encontradas com as tarefas do TEMPA envolvendo habilidades manuais finas, como manusear moedas e pegar e mover pequenos objetos $(r=.59$ e $r=.58$, respectivamente).

No presente estudo, os maiores valores de correlação foram verificados entre a destreza digital (NHPT) e a tarefa pegar e mover objetos pequenos do TEMPA nas mulheres. Nos homens foram verificados maiores valores de correlação entre esta tarefa e a destreza manual (BBT). Desrosiers et al. (1995) verificaram que os homens apresentam maior velocidade de execução nas tarefas do TEMPA que envolvem destreza manual e força, enquanto as mulheres são mais rápidas nas atividades que envolvem destreza digital. Como foi observado, a destreza manual e digital são parâmetros importantes na realização de tarefas manuais e assim, prérequisitos para um bom desempenho.

\section{CONCLUSÕES}

Foram estabelecidos os valores de referência da capacidade funcional para os membros superiores por meio do TEMPA para adultos e idosos do sul do Brasil, em função da faixa etária, sexo e lateralidade. Esses valores poderão servir de parâmetro para futuras pesquisas e intervenções na busca de minimizar os efeitos negativos do processo de envelhecimento e de patologias que comprometem a capacidade funcional dos membros superiores.

Os resultados sugerem que, com o avançar da idade, ocorre um declínio da capacidade funcional dos membros superiores, em ambos os sexos, que é mais evidente a partir dos 70 anos. Observou-se relação entre a destreza manual (BBT) e digital (NHPT) e a capacidade funcional dos membros superiores (TEMPA) em ambos os sexos, sendo que nas mulheres a correlação foi maior com a destreza digital e nos homens com a destreza manual. Os resultados sugerem que, além dos exercícios voltados para o fortalecimento muscular, sejam contempladas nos programas de intervenção atividades que possam manter/melhorar as habilidades manuais, respeitando as eventuais diferenças entre os sexos.

\section{REFERÊNCIAS}

Amirjani, N., Ashworth, N. L., Gordon, T., Edwards, D. C., \& Chan. K. M. (2007). Normative values and the effects of age, gender, and handedness on the Moberg pick-up test. Muscle \& Nerve, 35, 788-792. 
Desrosiers, J., Bravo, G., Hébert, R., Dutil, E., \& Mercier, L. (1994). Validation of the box and block test as a measure of dexterity of elderly people: Reliability, validity, and norms studies. Archives of Physical Medicine and Rehabilitation, 75, 751-755.

Desrosiers, J., Hébert, R., Bravo, G., \& Rochette, A. (1999). Age-related changes in upper extremity performance of elderly people: A longitudinal study. Experimental Gerontology, 34, 393-405.

Desrosiers, J., Hébert, R., Bravo, G., \& Dutil, E. (1995). Upper extremity performance test for the elderly (TEMPA): Normative data and correlates with sensorimotor parameters. Archives of Physical Medicine and Rehabilitation, 76, 1125-1129.

Desrosiers, J., Hébert, R., Dutil, E., \& Bravo, G. (1993). Development and reliability of an upper extremity function test for the elderly: The TEMPA. Canadian Journal of Occupational Therapy, 60(1), 9-16.

Geraldes, A. A. R., Oliveira, A. R. M., Albuquerque, R. B., Carvalho, J. M., \& Farinatti, P. T. V. (2008). A força de preensão manual é boa preditora do desempenho funcional de idosos frágeis: Um estudo correlacional múltiplo. Revista Brasileira de Medicina do Esporte, 14(1), 1216.

Hackel, M. E., Wolfe, G. A., Bang, S. M., \& Canfield, J. S. (1992). Changes in hand function in the aging adult as determined by the Jebsen test of hand function. Physical Therapy, 72(5), 373-377.

Instituto Brasileiro de Geografia e Estatística - IBGE (2002). Estudos e pesquisas informação demográfica e socioeconômica: Perfil dos idosos responsáveis pelos domicílios no Brasil, 2000. Rio de Janeiro: Autor.

Jebsen, R. H., Taylor, N., Trieschmann, R. B., Trotter, M. J., \& Howard, L. A (1969). An objective and standardized test of hand function. Archives of Physical Medicine and Rehabilitation, 50(6), 311-319.

Kellor, M., Frost, J., Silberberg, N., Iversen I., \& Cummings, R. (1971). Hand strength and dexterity: Norms for clinical use. American Journal of Occupational Therapy, 25, 77-83.

Mathiowetz, V., Volland, G., Kashman, N., \& Weber, K. (1985). Adult norms for the box and block test of manual dexterity. American Journal of Occupational Therapy, 39(6), 386-391.

Mathiowetz, V., Weber, K., Kashman, N., \& Volland, G. (1985). Adult norms for the nine hole peg test of finger dexterity. American Journal of Occupational Therapy, 5(1), 24-37.

Mendes, M. F., Tilbery, C. P., Balsimelli, S., Moreira, M. A., \& Cruz, A. M. B. (2001). Teste de destreza manual da caixa e blocos em indivíduos normais e em pacientes com esclerose múltipla. Arquivos de Neuro-Psiquiatria, 59(4), 889-894.

Michaelsen, S. M., Natalio, M. A., Silva, A. G., \& Pagnussat, A. S. (2008). Tradução e adaptação do TEMPA ('Test d'Évaluation des Membres supérieurs des Personnes Agées') para o português e validação para adultos portadores de hemiparesia. Revista Brasileira de Fisioterapia, 12(6), 511-519.

Polvin, A. R., Syndulcko, K., Tourtellote, W. W., Lemmon, J. A., \& Polvin, J. (1980). Human neurologic function and the aging process. Journal of American Geriatric Society, 28, 1-9.

Shiffman, L. M. (1992). Effects of aging on adult hand function. American Journal of Occupational Therapy, 46, 785-792.

Tilbery, C. P., Mendes, M. F., Thomaz, R. B., Oliveira, B. E. S., Kelian, G. L., Busch, R. B., ... Caleffi, P. (2005). Padronização da Multiple Sclerosis Functional Composite Measure (MSFC) na população brasileira. Arquivos de Neuro-Psiquiatria, 63(1), 127-132.

(c) EY-No quando especificado em contrário e nos conteúdos retirados de outras fontes bibliográficas. 